\title{
References
}

ANACH CENTER PUBLICATIONS, VOLUME

PWN-POLISH SCIENTIFIC PUBLISHERS

WARSAW 1979

[1] W. H. F l e m ing and R. W. R is he 1, Deterministic and stochastic optimal control, Appl. Math. 1, Springer-Verlag, 1975.

[2] B. I. Grigeli on is and A.N. Shiry a ev, On Stefan problem and optimal stopping rules for Markov processes, Theor. Probability Appl. 11 (1966), pp. 541-558.

[3] K. It o, Stochastic processes, Lect. Notes 16, Aarhus Univ., 1969.

[4] N.V. K r y lov, Control of a solution of a stochastic integral equation, Theor. Probability Appl. 17 (1972), pp. 114-131.

[5] -, On Bellman's equation, Proc. School-Seminar on the theory of random processes, Part I, Vilnius 1974, pp. 203-235.

[6] -, Optimal stopping of controlled diffusion processes, 3rd USSR-Japan Symp. Prob. Th., 1975.

[7] H. P. M c K e a n, Stochastic integrals, Academic Press, 1969.

[8] M. N i s i o, Some remarks on stochastic optimal controls, 3rd USSR-Japan Symp. Prob. Th. 1975.

[9] -, On a non-linear semi-group attached to stochastic optimal control, Publ. R.I.M.S. Kyoto Univ. 12 (1976), pp. 513-537.

[10] -, On stochastic optimal controls and envelope of Markovian semi-groups, Proc. Intern. Symp. SDE Kyoto 1976, pp. 297-325.

[11] K. R. Parthas arathy, Probability measures on metric spaces, Academic Press, 1967. [12] K. Y o s id a, Functional analysis, Springer-Verlag, 1968.

[13] J. Z a b c z y k, Optimal control by means of switchings, Studia Math. 45 (1973), pp. 161-171.

[14] -, Stochastic control with at most denumerable number of corrections, Proc. IFIP Conf., Rom 1974.

Presented to the Semester

Probability Theory.

February 11-June 11, 1976

\section{О МОМЕНТАХ ОСТАНОВКИ ПОЛУУСТОЙЧИВЫХ ДИФФУЗИОННЫХ ПРОЦЕССОВ}

\author{
А. А. НОВИков
}

Математический институт АН СССР, Москва, СССР

Рассматриваются неқоторые классы моментов остановки полуустойчивьх диффузионных процессов. Получены точные и асимптотические результаты, характеризующие распределения рассматриваемых моментов остановки.

\section{I. Введение}

Одномерньй марковский процесс $\left(X_{t}, \mathscr{F}_{t}, P_{x}\right)$ со значениями на полупрямой $R^{+}$называется полуустойчивым, если его переходная функция $P(t, x, B)$ $\left(B \in \mathscr{B}^{+}\right.$- борелевская $\sigma$-алгебра на $\boldsymbol{R}^{+}$) обладает автомодельным свойством, T.e.

$$
P(r t, X, B)=P\left(t, r^{-\alpha} x, r^{-\alpha} B\right)
$$

при всех $r>0$ и некотором показателе $\alpha>0$. Полуустойчивые марковские процессы на $\boldsymbol{R}^{+}$ввел и описал Ламперти в работе [5], в которой поқазано, что все невырожденные полуустойчивые диффузионные процессы порождаются оператором

$$
L_{x} f(x)=b x^{1-1 / \alpha} f^{\prime}(x)+d x^{2-1 / \alpha} f^{\prime \prime}(x),
$$

где $d>0$ и $b$ - параметры. Мы будем далее обозначать $\varrho=\frac{\alpha b}{d}+1-\alpha$. Из результатов Ламперти следует, что граница $x=\infty$ при всех значениях параметров является естественной, а граница $x=0$ является естественной только при $\varrho \geqslant 1 ;$ при $\varrho \geqslant 0$ является захватываюшей и при $0<\varrho<1$ является регулярной, причем в силу предположения о нетрерьпности траекторий на границе $x=0$ может быть либо поглощение либо отражение.

В этой заметке рассматриваются следующие классы моментов остановки:

$$
\begin{aligned}
\tau_{a} & =\inf \left\{t \geqslant 0: X_{t} \leqslant a(t+y)^{\alpha}\right\}, \\
\sigma_{c} & =\inf \left\{t \geqslant 0: X_{t} \geqslant c(t+y)^{\alpha} \text { или } X_{t}=0\right\}, \\
\sigma_{c} & =\inf \left\{t \geqslant 0: X_{t} \geqslant c(t+y)^{\alpha}\right\},
\end{aligned}
$$


при любьг значениях параметров $a \geqslant 0, y \geqslant 0$ и $c \geqslant 0$. Если $X_{0} \equiv x \leqslant a y^{\alpha}$, то, очевидно, $\tau_{\alpha}=0$, и если $x \geqslant c y^{\alpha}$, то $\sigma_{c}=\tilde{\sigma}_{c}{ }^{\circ}=0$. В дальнейшем мы будем предполагать вьполнение противоположжных неравенств. Из результатов Ламперти следует, что $P_{x}\left(\tau_{0}=\infty\right)=1$ при $\varrho \geqslant 1$ и $P_{x}\left(\tau_{0}<\infty\right)=1$ при $\varrho<1$ (в этом случае распределение $\tau_{0}$ вычисляется в явном виде, см. формулу (14)). Отсюда следует, что момент остановки $\tilde{\sigma}_{c}$ определен только при $\varrho \geqslant 1$.

Моменты остановки $\sigma_{c}$ и $\tilde{\sigma}_{c}$ рассматривались в работе Куджмы [3], в которой была решена задача об оптимальной остановке полуустойчивых дхффузионных процессов для цепи вида

$$
\operatorname{Sup}_{\tau \in M} E_{x} X_{\tau}^{\delta}(y+\tau)^{-\nu}, \quad \delta>0, \gamma>0,
$$

где $M$ - множество моментов остановки. В [3] найдень условия существования оптимального правила остановки для цепи указанного вида и показано, что при $\varrho \geqslant 1$ и при $0<\varrho<1$ с условием отражения в нуле оптимальное правило находится в классе моментов $\tilde{\sigma}_{c}$, а при $\varrho \leqslant 0$ и при $0<\varrho<1$ с условием поглощения в нуле - в классе $\sigma_{c}$. Куджма нашел уравнения для оптимальньх значений $c$ и показал, что оптимальные правила остановки конечны с вероятностью единица. Из формулируемьтх ниже результатов можжно тоже очевидным образом получить уравнение для оптимальныт значений $\dot{c}$, а также найти условия существования степенньх моментов оптимальныт правил остановки.

\section{II. Основные резулітаты}

Обозначим $\varkappa(\nu, \varrho)$ - наибольиий положительный корень функции $\Psi(-\nu, \varrho, z)$. и $\varkappa^{*}(v, \varrho)$ - наименьиий положительный корень $\Phi(-v, \varrho, z)$, где $\Psi(\cdot, \cdot, z)$ и $\Phi(\cdot, \cdot, z)-$ стандартные обозначения вырожденныт гитергеометрических функций (см., например, [1]). Функция $\Psi(-\nu, \varrho, z)$ не имеет положительных нулей только при $\varrho \geqslant 1$, когда $v<0$ и при $\varrho<1, v<1-\varrho$; в этих случаях нам будет удобно считать $\varkappa(v, \varrho)=0$. Еслщ $\varrho>0$, то функция $\Phi(-v, \varrho, z)$ не имеет положительных нулей только при $v<0$; в этом случае будем полагать $x^{*}(\nu, \varrho)=+\infty$.

УТВержДЕНИЕ 1. При всех $v, \varrho$ u $n p u \alpha^{2} a^{1 / \alpha} / d>x(v, \varrho)$

$$
E_{x}\left(\tau_{a}+y\right)^{v}=y^{\nu} \frac{\Psi\left(-v, \varrho, \alpha^{2} \chi^{1 / \alpha} / y d\right)}{\Psi\left(-v, \varrho, \alpha^{2} a^{1 / \alpha} / d\right)}
$$

Eслu $\alpha^{2} a^{1 / \alpha} / d \leqslant \varkappa(\nu, \varrho)$, mo $E_{x} \tau_{a}^{\nu}=\infty$.

УТВЕРЖДВНИЕ 2. При всех $v, \varrho<1 u n p u \alpha^{2} c^{1 / \alpha} d<\varkappa^{*}(\nu+\varrho-1,2-\varrho)$

(2) $\quad E_{x}\left(\sigma_{c}+y\right)^{\nu}=y^{\nu}\left(\frac{x^{1 / \alpha}}{c^{1 / \alpha} y}\right)^{1-e} \frac{\Phi\left(-\nu+\varrho-1,2-\varrho, \alpha^{2} x^{1 / \alpha} / y d\right)}{\Phi\left(-\nu+\varrho-1,2-\varrho, \alpha^{2} c^{1 / \alpha} / d\right)} \times$

$$
\times\left[1-\frac{\Psi\left(-v, \varrho, \alpha^{2} c^{1 / \alpha} / d\right)}{\Psi(-\nu, \varrho, 0)}\right]+y^{\nu} \frac{\Psi\left(-\nu, \varrho, \alpha^{2} x^{1 / \alpha} / y d\right)}{\Psi(-\nu, \varrho, 0)},
$$

где значения правой части при $v=1-\varrho+k, k=0,1,2, \ldots$, вычисляются по непрерывности. Если $\alpha^{2} c^{1 / \alpha} / d \geqslant x^{*}(\nu+\varrho-1,2-\varrho)$, то $E_{x} \sigma_{c}^{\nu}=\infty$.

УтвержДЕние 3. При всех $v, \varrho>0 u n p u \alpha^{2} c^{1 / \alpha} / d<x^{*}(\nu, \varrho)$

$$
E_{x}\left(\tilde{\sigma}_{c}+y\right)^{\nu}=y^{\prime \prime} \frac{\Phi\left(-\nu, \varrho, \alpha^{2} x^{1 / \alpha} / y d\right)}{\Phi\left(-\nu, \varrho, \alpha^{2} c^{1 / \alpha} / d\right)} .
$$

$E c л u \alpha^{2} c^{1 / \alpha} / d \geqslant x^{*}(\nu, \varrho)$, mo $E_{x} \tilde{\sigma}_{c}^{\prime \prime}=\infty$.

Метод, которым получены эти формулы, состоит в следующем. Мы подбираем специальным образом функцию $U(t, x)$ такую, что троцесс $U(t \wedge$ $\left.\wedge \tau, X_{t \wedge \tau}\right)$, где $\tau$ - некоторый момент остановки ил $t \wedge \tau=\min (t, \tau)$ явля-

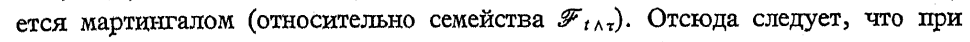
некоторых условиях на момент остановки $\tau$ выполняется соотношение $E_{x} U\left(\tau, X_{\tau}\right)=U(0, x)$. Из этого соотношения иі выводятся затем нужные формулы. Подобный метод использовался во многих работах. Например, в работе ШІетта [8] аналогичным методом найдены моменты времени первого выхода винеровского продесса на двухстороннюю границу параболичесқого типа (этот результат является частным случаем утверждения 3 при $\bar{x}=0$, $\alpha=1 / 2, b=0, d=1)$. Подобный метод использовался в работе [6], а также в работах [7] и. [4] для расчета вероятностей пересечения границ, зависящих от временх.

\section{III. Доказательство утверждений 1,2 и 3}

Полуустойчивый диффузионный процесс $X_{t}$ можно рассматривать при $t<\xi_{\varepsilon}$, $\xi_{\varepsilon}=\inf \left\{t \geqslant 0: X_{t}=\varepsilon\right\}, \quad \varepsilon>0$, қак процесс, ушравляемый стохастическим уравненхем

$$
X_{t}=x+\int_{0}^{t} B\left(X_{s}\right) d s+\int_{0}^{t} \sigma\left(X_{s}\right) d w_{s}
$$

где $w_{s}$ - стандартный винеровский процесс, $B(x)=b x^{1-1 / \alpha}$ ил $\sigma(x)=\sqrt{2 d} x^{1-1 / \alpha}$ при $x<\varepsilon$ и соответствующим образом доотределяются при $x \leqslant \varepsilon$ (см. [2], гл. 3). Пусть функция $U(t, x)$ дважды дифференцируема по $x$ при $x>0$, жепрерывно дифференцируема по $t$ и удовлетворяет уравнению

$$
U_{t}^{\prime}(t, x)+L_{x} U(t, x)=0 .
$$

Тогда, применяя формулу Ито ([8]) к $U\left(t, X_{t}\right)$, получим, что для любого момента остановки $\tau\left(\tau \leqslant \xi_{\varepsilon}\right)$

$$
U\left(\tau, X_{\tau}\right)=U(0, x)+\int_{0}^{\tau} \sqrt{2 d} X_{s}^{1-1 / 2 \alpha} U_{x}^{\prime}\left(s, X_{s}\right) d w_{s} .
$$

Математическое оясидание стохастического интеграла в этом соотношении равно нулюо при вьтолнених, например, следующщего условия ([б)

$$
E_{x}\left(\int_{0}^{\tau} X_{s}^{2-1 / \alpha}\left[U_{x}\left(s, X_{s}\right)\right]^{2} d s\right)^{1 / 2}<\infty
$$

(при этом условии процесс $U\left(t \wedge \tau, X_{t \wedge \tau}\right.$ ) является мартингалом). 
Пусть далее $U\left(t, X_{t}\right)=(t+y)^{y} \varphi\left(z_{t}\right)$, где $z_{t}=\alpha^{2} X_{t}^{1 / \alpha} /(t+y) d, y>0$. Тогда из (5) получим следующее уравнение для $\varphi(z)$ :

$$
z \frac{d^{2}}{d z^{2}} \varphi(z)+(\varrho-z) \frac{d}{d z} \varphi(z)+\nu \varphi(z)=0 .
$$

Решениями этого уравнения являются функции

(9) $\Psi(-v, \varrho, z), z^{1-\varrho} \Phi(-v+\varrho-1,2-\varrho, z)(\varrho<1)$

$$
\text { is } \Phi(-\nu, \varrho, z)(\varrho \neq 0,-1,-2, \ldots) \text {, }
$$

из которьх можно составить фундаментальную систему решеници, причем эти функции являются аналитичесқими функциями от $\nu$ при действительных $\varrho$ и $z \geqslant 0$ (для қоторых они определены) (см. [1]).

Приступим теперь непосредственно к выводу формул (1) и (2). Положим в (6) $\tau=\sigma_{c} \wedge \tau_{a}, a>0$. Ясно, что при достаточно мальх $\varepsilon>0$ п.в, $\tau_{a} \leqslant \xi_{\varepsilon}$. Нетрудно проверить, что в силу неравенств $0<a \leqslant X_{S}(s+y)^{-\alpha} \leqslant c$ при всех $s \leqslant \sigma_{c} \wedge \tau_{a}$, условие (7) заведомо выполняется при $\nu<0$ для любой функціии из набора (9) (при $v>0$ выполнено, если $E_{x}\left(\sigma_{c} \wedge \tau_{a}+y\right){ }^{y}<\infty$ ). Поэтому из (6) получаем, учитывая непрерывность траекторищ $X_{t}$, что для любого решения уравнения (8) при $\nu<0$

$$
\begin{array}{r}
E_{x} I\left(\sigma_{c} \leqslant \tau_{a}\right)\left(\sigma_{c}+y\right)^{\prime} \varphi\left(\alpha^{2} c^{1 / \alpha} / y\right)+E_{x} I\left(\tau_{a}<\sigma_{c}\right)\left(\tau_{a}+y\right)^{\prime \prime} \varphi\left(\alpha^{2} a^{1 / \alpha} / y\right)= \\
=y^{y} \varphi\left(\alpha^{2} x^{1 / \alpha} / y d\right),
\end{array}
$$

где $I($ ) - индиқаторная функция.

Теперь вывод формул (1) и (2) при $v<0$ сводится қ подбору решений уравнения (8) и предельным переходам. Рассмотрим в качестве $\varphi(z)$ функцию $\Psi(-\nu, \varrho, z)$. Известно, что

$$
\Psi(-\nu, \varrho, z)=z^{v}\left(\left(1+O\left(|z|^{-1}\right)\right), \quad z \rightarrow \infty,\right.
$$

(см. [1], это единственное с точностью до константы решение уравнения (8), имеющее степенную асимптотику на бесконечности). Учитывая этот факт, нетрудно поқазать, что предел первого слагаемого в (10) при $c \rightarrow \infty$ равен нулю. Предельный переход во втором слагаемом дает формулу (1) при всех $\nu<0$

Для доказательства этой формуль при $\nu \geqslant 0$, заметим, ччо правая часть формулы представляет отношение двух аналитических функций. Поэтому правая часть формулы (1) представляет аналитическую функико от $v$ при всех $\nu$ из области $\operatorname{Re} \nu<\bar{\nu}$, где $\bar{\nu}$ - определяется из равенства $\alpha^{2} a^{1 / \alpha} / d=$ $=x(\nu, \varrho)$. Из этого фақта следует возможность аналитического продолжения равенства в формуле (1) на все $\nu$, определяемьгх из условия $\alpha^{2} a^{1 / \alpha} / d>$ $>x(\nu, \varrho)$.

Для доказательства формулы (2) рассмотрим в качестве $\varphi(z)$ функсиии $\Psi(-\nu, \varrho, z)$ и $z^{1-\varrho} \Phi(-\nu-\varrho+1,2-\varrho, z)(\varrho<1)$
Для этих функций предельным переходом при $a \rightarrow 0$ в (10) с учетом того, что $\Phi(-v, \varrho, 0)=1$, получим

$$
\begin{aligned}
& E_{x} I\left(\sigma_{c}<\tau_{0}\right)\left(\sigma_{c}+y\right)^{\nu} \Psi\left(-\nu, \varrho, \alpha^{2} c^{1 / \alpha} / d\right)+E_{x} I\left(\sigma_{c}=\tau_{0}\right)\left(\sigma_{c}+y\right)^{\nu} \Psi(-\nu, \varrho, 0)= \\
& =y^{\nu} \Psi\left(-\nu, \varrho, \alpha^{2} x^{1 / \alpha} / d\right) \text {, } \\
& E_{x} I\left(\sigma_{c}<\tau_{0}\right)\left(\sigma_{c}+y\right)^{\nu}\left(\frac{\alpha^{2}}{d} c^{1 / \alpha}\right)^{1-e} \Phi\left(-\nu+\varrho-1,2-\varrho, \frac{\alpha^{2}}{d} c^{1 / \alpha}\right)= \\
& =y^{\nu}\left(\frac{\alpha^{2}}{d} x^{1 / \alpha}\right)^{1-\varrho} \Phi\left(-\nu+\varrho-1,2-\varrho, \frac{\alpha^{2}}{d} x^{1 / \alpha}\right) \text {. }
\end{aligned}
$$

Из этих соотношений получаем формулу (2) при всех $v<0$. Отметим, что

$$
\Psi(-v, \varrho, 0)=\Gamma(1-\varrho) / \Gamma(1-\varrho-v),
$$

где $\Gamma(z)$ - гамма-функция. Поэтому правая часть формулы (2) неопределена при $v=1-\varrho+k, k=0,1,2, \ldots$ Если $\varrho \neq 0,-1,-2, \ldots$, то эта неопределенность раскрывается с помощью известной формулы, связывающей функции $\Phi(-v, \varrho, z)$ х $\Psi(-v, \varrho, z)$ (см. [1], формула 6.5.7). Применяя эту формулу, мы получаем следующее выраженще для правой части (2) при $\varrho \neq 0,-1,-2, \ldots$

$$
\begin{aligned}
& y^{\nu}\left(\frac{x^{1 / \alpha}}{c^{1 / \alpha} y}\right)^{1-\varrho} \frac{\Phi\left(-\nu+\varrho-1,2-\varrho, \alpha^{2} x^{1 / \alpha} / y\right)}{\Phi\left(-\nu+\varrho-1,2-\varrho, \alpha^{2} c^{1 / \alpha} / d\right)} \times \\
& \times\left[1-\Phi\left(-\nu, \varrho, \alpha^{2} c^{1 / \alpha} / d\right)\right]+y^{\nu} \Phi\left(-\nu, \varrho, \alpha^{2} x^{1 / \alpha} / y d\right) .
\end{aligned}
$$

Для раскрытия неогределенности в случае $\varrho=0,-1,-2, \ldots$ (так называемый ,логарифмический" случай) надо воспользоваться формулами (6.7.13) из [1] (мы не приводим эту формулу ввиду ее громоздкости). Во всех случаях мьг получаем, что правая часть формулы (2) является аналитической функцией от $\nu$, в области $\operatorname{Re} \nu<\nu^{*}$, где $\nu^{*}$ определяются из равенства $\alpha^{2} c^{1 / \alpha} / d=x^{*}(v+\varrho-1,2-\varrho)$. Отсюда следует утверждение 2 в общем случае.

Формулу (3) при всех $\varrho \geqslant 1$ можно получить тем же методом, что и выше были найдены формулы (1) и (2). Действительно, если рассмотреть в качестве $\varphi(z)$ функцию $\Phi(-\nu, \varrho, z)$, то с учетом того, что $P\left(\tau_{0}=\infty\right)=1$ при $\varrho \geqslant 1$, получим скачала (3) при $v<0$, а затем с помощью аналитичесқого продолжения. при всех $\nu$, определяемых из условия $\alpha^{2} c^{1 / \alpha} / d<\varkappa^{*}(\nu, \varrho)$. Случай $0<$ $<\varrho<1$ требует особого рассмотрения, так қақ в этом случае $P\left(\sigma_{c}>\tau_{0}\right)>0$, а описание процесса с помопиьо стохастичесқого уравнения (4) возможно только при $t<\tau_{0}$. В этом случае можно воспользоваться, например, результатами работы Лея [4], в которой обобщается известная формула Дьпқина [2] на случай функций, зависящих от времени. С помошью теоремь 1 этой работы можкно доказать, что процесс $\left(t \wedge \sigma_{c}+y\right)^{\nu} \Phi\left(-\nu, \varrho, \frac{\alpha^{2}}{d} x_{t \wedge \sigma_{c}}^{1 / \alpha}\right), \nu<0$, является мартингалом относительно $\mathscr{F}_{t \wedge \sigma_{c}}$. Доказательство этого фақта сводится к непосредственной проверке условий теоремы 1 из [4] для процесса 
$x_{t}$ с временем жизни $\xi=\sigma_{c}$ и функцией $\varphi(x)=1$ (в обозначениях этой теоремы). Отсода следует, что при $v<0$

(13) $E_{x}\left(t \wedge \sigma_{c}+y\right)^{v} \Phi\left(-v, \varrho, \frac{\alpha^{2}}{d} x_{t \wedge \sigma_{c}}^{1 / \alpha} /\left(t \wedge \sigma_{c}+y\right)\right)=y \Phi\left(-\nu, \varrho, \frac{\alpha^{2}}{y d} x\right)$.

Так как на множестве $\left\{t \leqslant \sigma_{c}\right\} x_{t}^{1 / \alpha} /(t+y) \leqslant c^{1 / \alpha}$ при всех $t$, то нетрудно проверить, что

$$
\lim _{t \rightarrow \infty} E_{x} I\left(t \leqslant \sigma_{c}\right) \Phi(-\nu, \varrho, \cdot)=0 .
$$

Поэтому из (13) предельным переходом по $t \rightarrow \infty$ получим формулу (3) при всех $\nu<0$. Далее, как обычпо, аналитичесқим продолжением получим формулу (3) и в общем случае.

\section{IV. Несколько следствй}

1. Из формулы (1) предельным переходом по $y \rightarrow 0$ в силу (11) получим

$$
E_{x} \tau_{a}^{\nu}=\left(\frac{\alpha^{2} x^{1 / \alpha}}{d}\right)^{\nu} \Psi^{-1}\left(-\nu, \varrho, \frac{\alpha^{2}}{d} a^{1 / \alpha}\right), \quad \frac{\alpha^{2}}{d} x^{1 / \alpha}>x(\nu, \varrho) .
$$

Так как при $\varrho<1$ выполняется (12), то еще одним предельным переходом по $a^{\prime} \rightarrow 0$ получаем

$$
E_{x} \tau_{0}^{\nu}=\left(\frac{\alpha^{2} x^{1 / \alpha}}{d}\right)^{\nu} \frac{\Gamma(1-\varrho)}{\Gamma(1-\varrho-\nu)}, \quad \varrho<1, \quad \nu<1-\varrho .
$$

Обращая это преобразование Меллина для $\tau_{0}$, находим, что

$$
\text { (14) } \frac{d}{d t} P_{x}\left(\tau_{0}<\frac{\alpha^{2}}{d} x^{1 / \alpha} t\right)=\frac{t^{\ell-2}}{\Gamma(1-\varrho)} \exp (-1 / t), \quad(t>0, x>0) \text {. }
$$

2. Формулы (1), (2) и (3) задают преобразование Лапласа для логарифмов рассматриваемых моментов остановки. Правые части этих формул являются аналитическими функциями от значений $\nu$, допускаемьг по условиям утверждений 1. Обозначим $\bar{\nu}(a)$ корень уравнения $x(\nu, \varrho)=\frac{\alpha^{2}}{d} a^{1 / \alpha}$ к $v^{*}(c, \varrho)$ корень уравнения $x^{*}(\nu, \varrho)=\frac{\alpha^{2}}{d} c^{1 / \alpha}$. Нетрудно проверить, что правая часть формульг (1) имеет простой полюс при $v=\bar{v}(a)$, а правые части формулы (2) и (3) имеет простые полюса при $\nu=\nu^{*}(c, 2-\varrho)+\varrho-1$ и $\nu=\nu^{*}(c, \varrho)$ соответственно. Поэтому применением теоремы тауберова типа получим, что при $t \rightarrow \infty$ :

$$
\begin{aligned}
& P_{x}\left(\tau_{a} \geqslant t\right)=c t^{-\bar{\nu}(a)}(1+o(1)), \\
& P_{x}\left(\sigma_{c} \geqslant t\right)=c t^{\rho-1-\nu *(c, 2-\varrho)}(1+o(1)), \quad \varrho<1, \\
& P_{x}\left(\tilde{\sigma}_{c} \geqslant t\right)=c t^{-r^{*}(c, \varrho)}(1+o(1)), \quad \varrho>0 .
\end{aligned}
$$

где константы $c$ зависят только от параметров процесса и моментов остановки.
3. Из конкретных результатов, полученных в этой работе, можно извлечь и некоторые общие результаты. Напрхмер, нетрудно доказать, что, если $x_{t}$ - полуустойчивый процесс с параметрами $\alpha$ и $\varrho(\varrho \geqslant 1)$ и $\tau=$ $=\inf \left\{t \geqslant 0: x_{t} \geqslant f(t)\right\}$, где $x<f(0), f(t)$ - непрерывная монотонная функция такая, что $f(t) t^{-\alpha}=o(1)$ при $t \rightarrow \infty$, то $E_{x} \tau^{\nu}<\infty$ при всех $\nu \geqslant 0$.

\section{Литература}

[1] Г. Б е й т м ан, А. Э р д е йн, Высшие трансуендентные функиии, Наука, Москва 1973.

[2] Е. Б. Д бг ни ки и, Марковские прочессьи, Физматгиз, Москва 1963.

[3] Р. А. К у дж м а, Оптимальная остановка полуустойчивых диффузионных проиессов, Литовск. мат. сб. 12 (1972), стр. 4-11.

[4] [T. Л. Л е 迆 T. L. L a i, Martingales and boundary crossing probabilities for Markov processes, Ann. Prob. 2 (1974), cтp. 1152-1168.

[5] [Дж. Л а м п е р т и] J. L a m perti, Semi-stable Markov processes, $I, \mathrm{Z}$. Wahrscheinlichkeitstheorie und Verw. Gebiete 223 (1972), crp. 205-225.

[6] А. А. Н о в ик о в, О моментах остановки винеровского прочесса, Теория вероятн. и её прим. 16 (1971), стр. 458-465.

[7] [Г. Р о б б и н с, Д. 3 и г м унд] H. R о b b ins, D. S i e g mund, Boundary crossing probabilities for the Wiener processes and sample sums, Ann. Math. Statist. 41 (1970), crp. 14101429.

[8] [Л. A. Ш $\mathrm{e} \mathrm{m} \mathrm{m}]$ L. A. S h e p p, A first passage problem for the Wiener process, Ann. Math. Statist. 38 (1967), crp. 1912-1914.

Presented to the Semester Probability Theory

February 11-June 11, 1976 